Check for updates

Cite this: RSC Adv., 2018, 8, 42025

\title{
Synthesis of anti-perovskite-type carbides and nitrides from metal oxides and melamine
}

\author{
Daigorou Hirai, (D)* Hidetake Tanaka, Daisuke Nishio-Hamane and Zenji Hiroi \\ Four anti-perovskite-type compounds, $\mathrm{ZnNNi}_{3}, \mathrm{ZnCNi}_{3}, \mathrm{SnNCO}_{3}$, and $\mathrm{SnCCO}_{3}$, are synthesised through \\ reactions between metal oxides and organic compound melamine $\left(\mathrm{C}_{3} \mathrm{H}_{6} \mathrm{~N}_{6}\right)$. $\mathrm{ZnNNi}_{3}$ and $\mathrm{ZnCNi}_{3}$ are \\ selectively synthesised by choosing different reaction temperatures and nominal oxide-to-melamine \\ ratios. $\mathrm{SnNCO}_{3}$ is synthesised for the first time by this melamine method. Resistivity, magnetisation, and \\ heat capacity measurements reveal that $\mathrm{SnNCO}_{3}$ is a correlated metal with a high density of states at the \\ Fermi level. The results demonstrate that this feasible synthetic route using melamine is useful in the \\ search for complex metal carbides and nitrides toward novel functional materials.
}

Received 12th September 2018

Accepted 11th December 2018

DOI: $10.1039 / \mathrm{c} 8 \mathrm{ra0} 07581 \mathrm{f}$

rsc.li/rsc-advances

anti-perovskite-type compounds sometimes suffer from a defi-

\section{Introduction}

Anti-perovskite-type compounds with a general formula of $\mathrm{AXM}_{3}(\mathrm{~A}=\mathrm{Ga}, \mathrm{Al}, \mathrm{In}, \mathrm{Zn}, \mathrm{Sn}$, etc.; X $=\mathrm{C}, \mathrm{N}, \mathrm{B} ; \mathrm{M}=$ transition metal) have been intensively studied owing to their unique physical properties and functionalities such as superconductivity, ${ }^{1-3}$ negative thermal expansion, ${ }^{4-7}$ giant magnetoresistance, ${ }^{8-10}$ magnetostriction effect, ${ }^{11,12}$ magnetocaloric effect, ${ }^{13-15}$ thermoelectric properties, ${ }^{16,17}$ and temperature coefficient of resistivity of approximately zero. ${ }^{18}$ As illustrated in Fig. 1(a), the crystal structure is closely related to the perovskite structure with the formula of $\mathrm{AMX}_{3}$. In the perovskite structure, the $\mathrm{A}$ and $\mathrm{M}$ sites are occupied by cations, where the $\mathrm{M}$ cation is octahedrally coordinated by six anions at the $\mathrm{X}$ sites, while in the anti-perovskite structure, an anion occupying the $\mathrm{X}$ site is surrounded by six $\mathrm{M}$ cations to form an $\mathrm{XM}_{6}$ octahedron. Many anti-perovskite-type compounds have been synthesised. However, considering the flexibility of the anti-perovskite-type structure, we believe that there are more compounds of this type that have not been yet studied.

Most of the anti-perovskite-type nitrides were synthesised by reaction of metal oxides with ammonia $\left(\mathrm{NH}_{3}\right)$ gas at high temperatures, typically approximately $800{ }^{\circ} \mathrm{C}$, which is usually referred to as ammonolysis. ${ }^{19}$ The reaction is driven by the high nitriding activity of ammonia; the high temperature is required to accelerate the solid-state reaction with the oxides. However, as ammonia is decomposed into chemically inactive nitrogen and hydrogen gases at above $300{ }^{\circ} \mathrm{C},{ }^{19}$ the actual ammonolysis is performed under a non-equilibrium condition in a bulk flow of ammonia gas during the decomposition process. Such a nonequilibrium reaction is difficult to control, and it is often difficult to obtain the desired nitride in a single phase. In particular,

Institute for Solid State Physics, University of Tokyo, Kashiwa, Chiba 277-8581, Japan. E-mail: dhirai@issp.u-tokyo.ac.jp ciency in the $\mathrm{X}$ site and mixing between the metal atoms, as observed in $\mathrm{GaC}_{1-x} \mathrm{Mn}_{3}$ (ref. 20) and $\mathrm{Sn}_{x} \mathrm{NFe}_{4-x}{ }^{21}$ Therefore, a synthetic method with high controllability is needed to produce stoichiometric anti-perovskite-type compounds.

Recently, new synthetic routes through reactions between organic reagents and metal oxides have been intensively investigated to overcome the difficulties of non-equilibrium gas-solid reactions. ${ }^{22-26}$ In the synthesis of nitrides, amide $\left(\mathrm{NaNH}_{2}\right),{ }^{27}$ azide $\left(\mathrm{NaN}_{3}\right),{ }^{28}$ and urea $\left(\mathrm{CO}\left(\mathrm{NH}_{2}\right)_{2}\right)^{29-32}$ have been used as nitriding reagent. As the reaction is performed in an

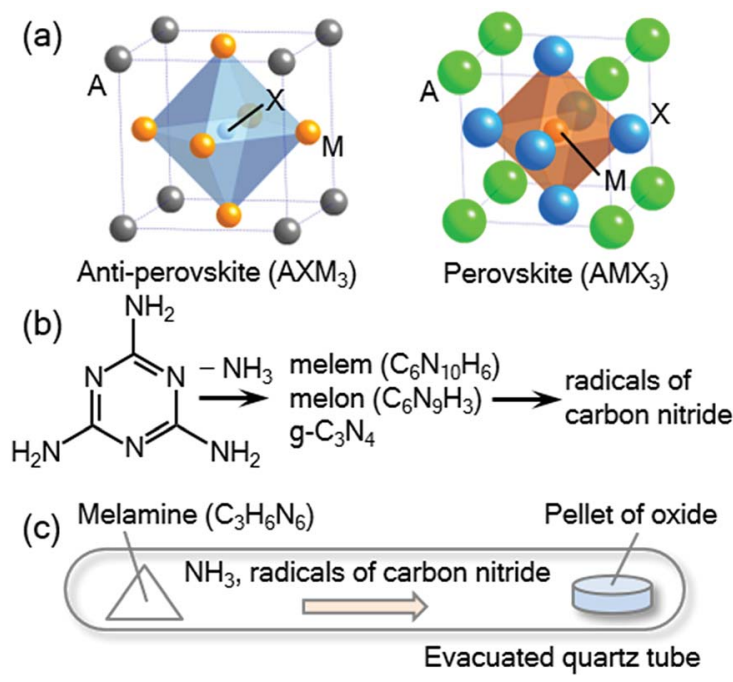

Fig. 1 (a) Crystal structures of the anti-perovskite-type compound $A_{X} M_{3}$ and related perovskite $A M X_{3}$. (b) Melamine $\left(\mathrm{C}_{3} \mathrm{H}_{6} \mathrm{~N}_{6}\right)$ gradually decomposes upon heating, releasing the reactive $\mathrm{NH}_{3}$ gas, which is decomposed into radicals of carbon nitrides. (c) Reaction tube. $\mathrm{NH}_{3}$ and radicals of carbon nitride from melamine react with oxides to generate an anti-perovskite-type nitride or carbide. 
enclosed tube, the ratio of reactant and nitriding reagent can be precisely controlled. Therefore, the reaction is highly reproducible compared to ammonolysis.

In this study, we focus on reactions using melamine $\left(\mathrm{C}_{3} \mathrm{H}_{6} \mathrm{~N}_{6}\right),{ }^{33}$ a widely used raw material that is chemically stable in air at ambient temperature. ${ }^{34}$ Binary nitrides of eight elements, Ga, Cr, B, Al, Ti, V, Nb, and Ta, were successfully prepared by reaction between melamine and oxide precursors. ${ }^{33}$ Upon heating, melamine decomposes into $\mathrm{g}-\mathrm{C}_{3} \mathrm{~N}_{4}$ releasing ammonia gas $\left(\mathrm{NH}_{3}\right)$ below $560{ }^{\circ} \mathrm{C}$. Further heating leads to the decomposition of $\mathrm{g}-\mathrm{C}_{3} \mathrm{~N}_{4}$ into reactive radical species containing hydrogen or carbon (Fig. 1(b)). ${ }^{35-37}$ It is considered that these reactive radicals form thermodynamically stable $\mathrm{CO}$ and $\mathrm{H}_{2} \mathrm{O}$, which promote the nitriding reactions. The above nitrides can be synthesised by the melamine method at significantly lower temperatures compared to that of conventional ammonolysis. It is worth noting that at higher temperatures and higher nominal melamine contents, binary carbides of Mo, W, V, Nb, and Ta were synthesised by the melamine method. ${ }^{38}$ This promising synthetic route may be applied to more complex compounds, which could reveal new compounds with novel functionalities.

In this study, we report the synthesis of anti-perovskite-type nitrides and carbides through reactions between the ingredient metal oxides and melamine, and their physical properties. $\mathrm{ZnNNi}_{3}$ and $\mathrm{ZnCNi}_{3}$ are selectively synthesised at different reaction temperatures and nominal melamine contents. A new anti-perovskite-type compound $\mathrm{SnNCo}_{3}$ is successfully synthesised, which is a correlated metal with Curie-Weiss-type magnetic susceptibility and large electronic specific heat coefficient, as revealed by heat capacity measurements. Our findings demonstrate that the melamine method is applicable to not only simple binary systems but also complex systems. The reaction using melamine is valuable for further studies on novel functional nitrides and carbides.

\section{Experimental}

In a typical synthesis, powders of ingredient oxides were mixed in an agate mortar and pressed into a pellet. The pellet was placed at one side of an evacuated fused-silica tube (typically $70 \mathrm{~mm}$ in length, $12 \mathrm{~mm}$ in inner diameter, and $14 \mathrm{~mm}$ in outer diameter) with melamine on the other side, as shown in Fig. 1(c). The relative amount of melamine was varied as listed in Table 1. The fused-silica tube was heated at reaction temperature for 12 hours. The total amount of melamine was adjusted so that the pressure in the tube at the temperature was low enough to avoid an explosion.

X-ray powder diffraction (XRD) measurements were conducted in a RINT-2500 diffractometer (Rigaku) using $\mathrm{Cu}-\mathrm{K} \alpha$ radiation. The data was collected at $298 \mathrm{~K}$ over a $2 \theta$ range between 10 and $120^{\circ}$ with a step size of $0.02^{\circ}$. Lattice parameters and atomic displacement factors were refined by the Rietveld method using the FULLPROF program. The background was characterized using a six-coefficient polynomial function. The chemical composition of the products was characterized by means of JEOL IT-100 scanning electron microscope with energy dispersive X-ray analyzer (EDX). The settings of $15 \mathrm{kV}, 0.8$ $\mathrm{nA}$, and $1 \mu \mathrm{m}$ beam diameter were used, and the ZAF method was employed for corrections.

Magnetization measurements were performed in the Magnetic Property Measurement System MPMS3 (Quantum Design) while resistivity and heat capacity measurements were conducted in the Physical Property Measurement System PPMS (Quantum Design).

\section{Results and discussion}

\section{Selective syntheses of $\mathrm{ZnNNi}_{3}$ and $\mathrm{ZnCNi}_{3}$}

We applied the melamine method to prepare the known antiperovskite-type nitride and carbide $\mathrm{ZnXNi}_{3}(\mathrm{X}=\mathrm{C}$ and $\mathrm{N})$. In previous reports, melamine and oxides were mixed into a pellet, ${ }^{33,38}$ in this study, however, a pelletised oxide was placed separately from melamine in an evacuated fused-silica tube so that gas-phase products from the decomposition of melamine react with the oxides. This method is suitable for measurements of physical properties as it reduces the contamination of products by decomposition residuals.

Three fabrications were performed with a fixed stoichiometric metal ratio $(\mathrm{ZnO}: \mathrm{NiO}=1: 3)$ at different melamine contents and reaction temperatures for $12 \mathrm{~h}$. The product obtained in condition 1 with a $\mathrm{ZnO}: \mathrm{NiO}: \mathrm{C}_{3} \mathrm{~N}_{3}\left(\mathrm{NH}_{2}\right)_{3}$ ratio of $1: 3: 3$ at $600{ }^{\circ} \mathrm{C}$ exhibits the XRD pattern shown in Fig. 2(a). All of the diffraction peaks were indexed to a cubic lattice at extinctions consistent with the space group $P m \overline{3} m$, which indicates that the product was synthesised in a single phase. The lattice constant of the product is $a=3.7648$ (3) $\AA$, which is comparable to that of the anti-perovskite-type nitride $\mathrm{ZnNNi}_{3}(\operatorname{Pm} \overline{3} m)$ of $a=3.756 \AA^{2}$. Other candidates were alloys of $\mathrm{Ni}$ and $\mathrm{Zn}$, and carbides and nitrides of $\mathrm{Ni}$ or Zn. However, they did not exhibit equal or similar XRD patterns to that in the figure. Therefore, we can conclude that $\mathrm{ZnNNi}_{3}$ was successfully synthesised in condition 1 .

Table 1 Starting composition, reaction temperature, products, and lattice parameters for the anti-perovskites synthesised in this study

\begin{tabular}{lclll}
\hline Starting composition & Temperature $\left({ }^{\circ} \mathrm{C}\right)$ & Products & Lattice parameter \\
\hline $\mathrm{ZnO}+3 \mathrm{NiO}+3 \mathrm{C}_{3} \mathrm{H}_{6} \mathrm{~N}_{6}$ & 600 & $\mathrm{ZnNNi}_{3}$ & $3.7648(3)$ & Reported values \\
$\mathrm{ZnO}+3 \mathrm{NiO}+5 \mathrm{C}_{3} \mathrm{H}_{6} \mathrm{~N}_{6}$ & 650 & $\mathrm{ZnCNi}_{3}$ & $3.6601(7)$ & 3.756 \\
$\mathrm{ZnO}+3 \mathrm{NiO}+5 \mathrm{C}_{3} \mathrm{H}_{6} \mathrm{~N}_{6}$ & 600 & $\mathrm{ZnNNi}_{3}$ & $3.7559(4)$ & 3.66 \\
& & $\mathrm{ZnCNi}_{3}$ & $3.6601(6)$ & 3.756 \\
$\mathrm{SnO}_{2}+3 \mathrm{CoO}+5 \mathrm{C}_{3} \mathrm{H}_{6} \mathrm{~N}_{6}$ & 1000 & $\mathrm{SnCCo}_{3}$ & $3.8118(3)$ & 3.66 \\
$\mathrm{SnO}_{2}+3 \mathrm{CoO}+5 \mathrm{C}_{3} \mathrm{H}_{6} \mathrm{~N}_{6}$ & 550 & $\mathrm{SnNCo}_{3}$ & $3.8513(1)$ & -
\end{tabular}




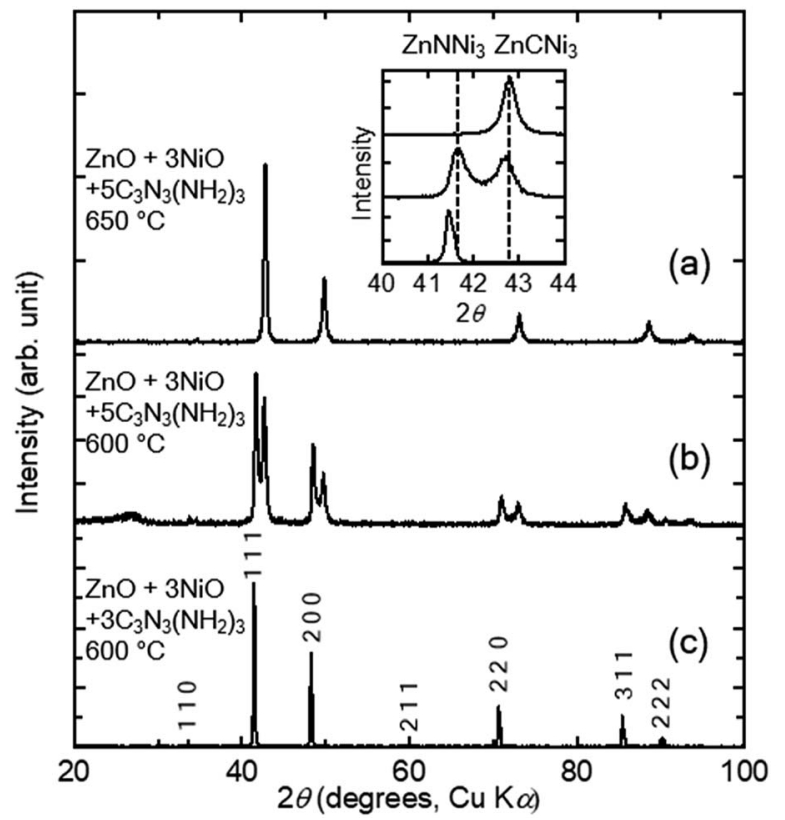

Fig. 2 Powder XRD patterns of the products from $\mathrm{ZnO}, \mathrm{NiO}$, and melamine prepared under different conditions. Monophasic samples of $\mathrm{ZnNNi}_{3}$ in condition 1 (a) and $\mathrm{ZnCNi}_{3}$ in condition 3 (c), and their mixtures in condition 2 (b) are obtained. The top inset presents an enlarged view showing differences in peak position between the three samples. The two broken lines represent the peak positions for $\mathrm{ZnNNi}_{3}$ and $\mathrm{ZnCNi}_{3}$.

In condition 2, the nominal melamine content was increased, yielding a $\mathrm{ZnO}: \mathrm{NiO}: \mathrm{C}_{3} \mathrm{H}_{6} \mathrm{~N}_{6}$ ratio of $1: 3: 5$; the reaction was performed at $600{ }^{\circ} \mathrm{C}$ for $12 \mathrm{~h}$. The XRD pattern of the product in Fig. 2(b) is different from that in condition 1. In addition to the peaks from $\mathrm{ZnNNi}_{3}$ obtained in condition 1, a set of peaks with similar relative intensities appeared at higher angles. This indicates that two phases with similar cubic structures and different lattice constants exist in the sample. The obtained lattice constants are $a=3.7559(4) \AA$, which corresponds to $\mathrm{ZnNNi}_{3}$, and $a=3.6601(6) \AA$.

In condition 3 , the nominal melamine content was equal to that in condition $2\left(\mathrm{ZnO}: \mathrm{NiO}: \mathrm{C}_{3} \mathrm{H}_{6} \mathrm{~N}_{6}=1: 3: 5\right)$, while the reaction temperature was higher $\left(650{ }^{\circ} \mathrm{C}\right)$. The XRD pattern of the product in Fig. 2(c) contains peaks corresponding only to those from the second cubic phase obtained in condition 2 with the smaller lattice constant. Therefore, an almost single cubic phase with lattice constant of $a=3.6601(7) \AA$ was obtained in condition 3. The lattice constant is $a=3.66 \AA$ for the antiperovskite-type carbide $\mathrm{ZnCNi}_{3}(P m \overline{3} m){ }^{39}$ no other known products reproduced the XRD pattern. Therefore, $\mathrm{ZnCNi}_{3}$ is synthesised by the melamine method at an excess melamine content and high reaction temperature.

The products and synthetic conditions are summarised in Table 1 . Ternary $\mathrm{Zn}-\mathrm{Ni}$ carbides and nitrides were selectively synthesised by the melamine method. The reaction mechanism will be discussed below. As complete phase separation was observed in intermediate condition 2 , there must be a miscibility gap between them in this synthetic condition. The lattice constants of the two phases in condition 2 are almost equal to those of the pure carbide and nitrides.

\section{Syntheses of a new nitride $\mathrm{SnNCo}_{3}$ and related carbide} $\mathrm{SnCCo}_{3}$

We applied our melamine method to search for new antiperovskite-type compounds and successfully obtained the nitride $\mathrm{SnNCo}_{3}$ and related carbide $\mathrm{SnCCo}_{3}$. In a similar reaction protocol, melamine and a pelletised mixture of $\mathrm{SnO}_{2}$ and $\mathrm{CoO}$ were sealed in an evacuated fused-silica tube at a molar ratio of $\mathrm{SnO}_{2}$ : $\mathrm{CoO}: \mathrm{C}_{3} \mathrm{H}_{6} \mathrm{~N}_{6}=1: 3: 5$ and heated at $550{ }^{\circ} \mathrm{C}$ for $12 \mathrm{~h}$ (condition 4). A single-phase compound crystallised in a cubic symmetry $(P m \overline{3} m)$ was obtained, as shown in the powder XRD pattern in Fig. 3(a). When the reaction temperature was increased to $1000{ }^{\circ} \mathrm{C}$ with the same nominal composition (condition 5), a compound with the same cubic symmetry but smaller lattice constant was obtained in a single phase. The lattice constants are $a=3.8513(1) \AA$ and $a=3.81181(3) \AA$ for conditions 4 and 5, respectively. The XRD pattern of the product in condition 5 is very similar to that of the reported antiperovskite-type carbide $\mathrm{SnCCo}_{3}$, while the lattice constant is close to that of the stoichiometric $\mathrm{SnCCo}_{3}$ of $a=3.8047(3) \stackrel{\AA}{{ }^{16}}{ }^{16}$ and larger than that of $\mathrm{SnCCO}_{3}$ with a carbon deficiency of $a=$ $3.78 \AA{ }^{40}$ Therefore, $\mathrm{SnCCo}_{3}$ with a minimum number of carbon defects was synthesised in this study.
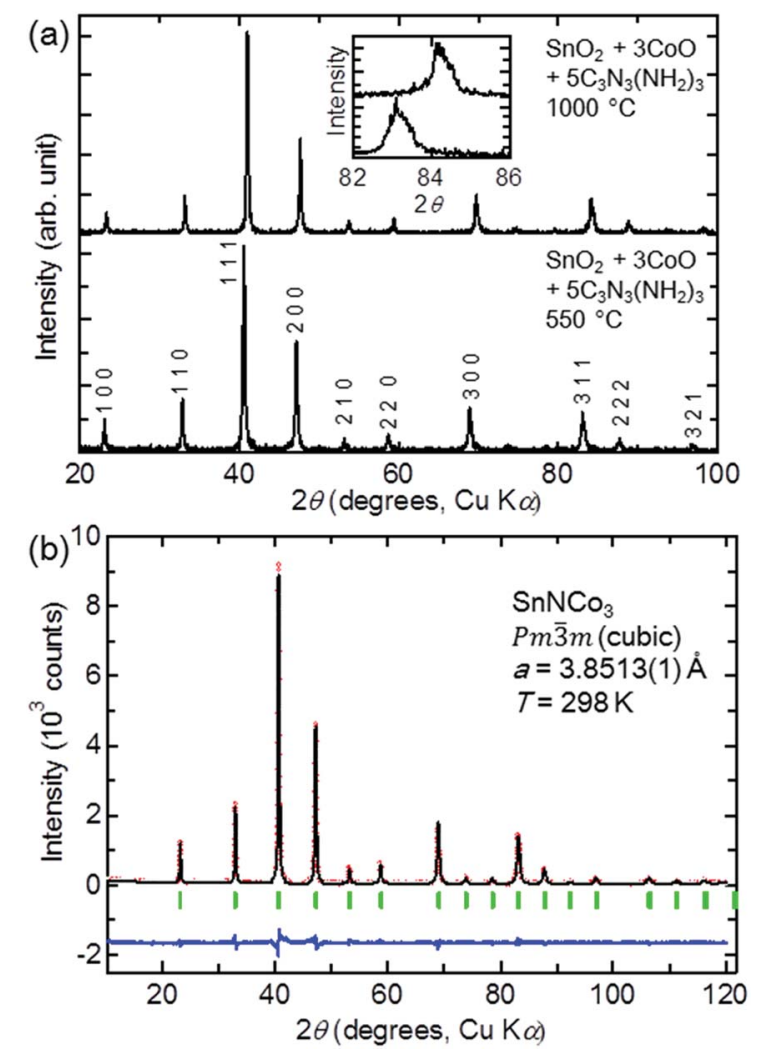

Fig. 3 (a) Powder XRD patterns of $\mathrm{SnNCO}_{3}$ and $\mathrm{SnCCO}_{3}$ prepared under conditions 4 and 5 by the melamine method, respectively. The inset shows an enlarged view of a $2 \theta$ range around the (311) diffraction peak. (b) Rietveld refinement for $\mathrm{SnNCO}_{3}$. The red circles, black solid line, blue solid line, and green tick marks represent experimental data, simulation pattern, differences, and diffraction positions, respectively. The atomic positions are: Sn $1 \mathrm{a}(000), \mathrm{Co} 3 \mathrm{c}(0) 0.50 .5)$, and N $1 \mathrm{~b}(0.5$ $0.50 .5)$. The Rietveld agreement factors are: $\chi^{2}=3.33, R_{\mathrm{Bragg}}=0.040$, $R_{\mathrm{P}}=0.086$, and $R_{\mathrm{WP}}=0.126$. 
The similar powder XRD patterns of the product in condition 4 suggest that another anti-perovskite-type compound was synthesised. Considering the lower reaction temperature and larger lattice constant, the product is likely $\mathrm{SnNCo}_{3}$. In order to confirm the formation of $\mathrm{SnNCO}_{3}$, the XRD pattern was fitted to a structural model based on the crystal structure of $\mathrm{SnCCO}_{3}$ by the Rietveld method. The fit was excellent, as shown in Fig. 3(b), although there are only a small number of refinable parameters with all of the atom positions fixed in the anti-perovskite-type structure. Therefore, we can conclude that a new anti-perovskite-type nitride $\mathrm{SnNCO}_{3}$ was obtained in condition 4 .

The lattice constants of $\mathrm{SnXCo}_{3}(\mathrm{X}=\mathrm{N}$ and $\mathrm{C})$ are larger than those of $\mathrm{ZnXNi}_{3}(\mathrm{X}=\mathrm{N}$ and $\mathrm{C})$. The metallic radii of the B-site cations Ni (1.149 $\AA)$ and Co $(1.157 \AA)$ are comparable to each other, while the radius of $\mathrm{Sn}(1.412 \AA)$ in the A-site is significantly larger than that of $\mathrm{Zn}(1.249 \AA) .{ }^{41}$ Therefore, the difference in lattice constant originates from the difference in size of the A-site ions. On the other hand, a comparison between the nitrides and carbides shows that the lattice constant of $\mathrm{ZnNNi}_{3}$ is $2.86 \%$ larger than that of $\mathrm{ZnCNi}_{3}$, while the difference between $\mathrm{SnNCo}_{3}$ and $\mathrm{SnCCO}_{3}$ is $1.04 \%$. These differences originate from the size difference between nitrogen and carbon atoms. In the case of $\mathrm{SnXCo}_{3}$, the effect of size difference seems to be suppressed in the expanded lattice by the large Sn cation.

\section{Chemical analysis}

The results of chemical analyses by EDX are shown in Fig. 4 . The atomic ratios of the metals were determined to be $\mathrm{Zn}: \mathrm{Ni}=$ $1: 3.04, \mathrm{Zn}: \mathrm{Ni}=1: 3.01, \mathrm{Sn}: \mathrm{Co}=1: 2.91$, and $\mathrm{Sn}: \mathrm{Co}=$ $1: 3.00$ for $\mathrm{ZnNNi}_{3}, \mathrm{ZnCNi}_{3}, \mathrm{SnNCo}_{3}$, and $\mathrm{SnCCO}_{3}$, respectively. The spectra confirm 1 to 3 ratio for $\mathrm{Zn}$ to $\mathrm{Ni}$ and $\mathrm{Sn}$ to Co in the products as expected for the anti-perovskites. The slightly reduced $\mathrm{Sn}$ : Co ratio from 3 in $\mathrm{SnNCo}_{3}$ may indicate off-stoichiometry, although the rough surface and small grain size of $\mathrm{SnNCo}_{3}$ may cause larger experimental error than for the others. Only a small amount of oxygen, typically less than $5 \%$ of the products, was detected in all the samples. This trace of oxygen could have originated from $\mathrm{O}_{2}$ and moisture absorbed on the surface of the sample and the silicon sample holder. Thus, the starting oxides completely reacted with $\mathrm{C}$ and $\mathrm{N}$ from melamine. For the carbide $\mathrm{ZnCNi}_{3}$ and $\mathrm{SnCCO}_{3}$ samples, no N element was detected. On the other hand, a significant amount of $\mathrm{C}$ is observed in all the sample, even for $\mathrm{ZnNNi}_{3}$ and $\mathrm{SnNCO}_{3}$. These carbon signals originated from the carbon tapes used for fixing the samples, by-product amorphous carbon covering the surface of sample particles, and contamination from the environment. Note that EDX measurement is not very sensitive to light elements such as $\mathrm{N}$ and C. A small amount of mixing between $\mathrm{N}$ and $\mathrm{C}$ or deficiencies may exist in the samples. Since the physical properties depend on the composition, it is highly desirable to quantify the $\mathrm{N}$ and $\mathrm{C}$ content by a different technique in future study.

\section{Physical properties of the new anti-perovskite-type nitride $\mathrm{SnNCo}_{3}$}

The physical properties of $\mathrm{SnNCo}_{3}$ were characterised by resistivity, magnetisation, and heat capacity measurements. As
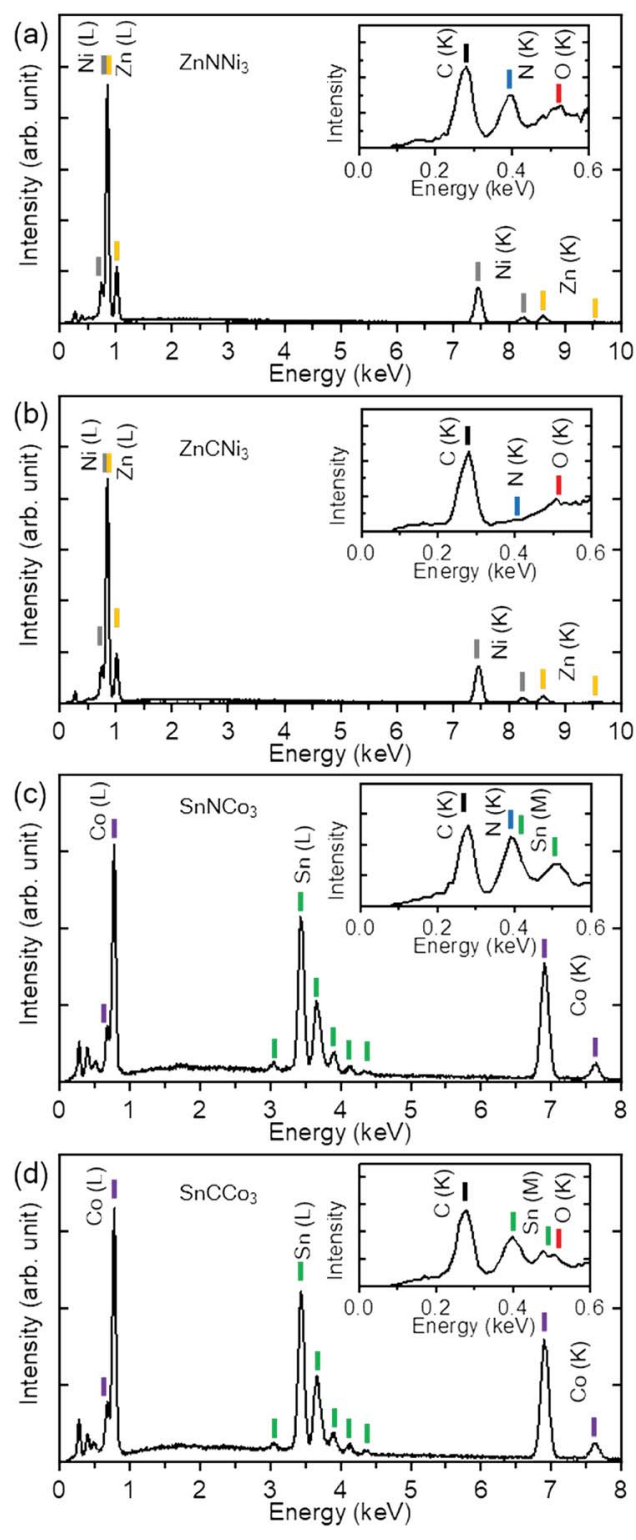

Fig. 4 Energy dispersive X-ray spectra for (a) $\mathrm{ZnNNi}_{3}$, (b) $\mathrm{ZnCNi}_{3}$, (c) $\mathrm{SnNCO}_{3}$, and (d) $\mathrm{SnCCO}_{3}$. The tick marks represent the positions of expected peaks. The insets present enlarged views of the low-energy region where the peaks from light elements appear.

shown in Fig. 5(a), our $\mathrm{SnCCo}_{3}$ sample exhibits metallic conductivity with temperature dependence and magnitude similar to those reported previously. ${ }^{16} \mathrm{SnNCo}_{3}$ also exhibits a metallic resistivity that is two orders of magnitude larger than that of $\mathrm{SnCCO}_{3}$, which may be attributed to grain boundary scattering as $\mathrm{SnNCO}_{3}$ was poorly sintered at lower temperature. A small upturn was observed below $25 \mathrm{~K}$ in the resistivity of $\mathrm{SnNCO}_{3}$, which may be due to weak localisation by the certain disorder. The observed metallic behaviour should be intrinsic considering that all related anti-perovskite-type compounds including $\mathrm{ZnXNi}_{3}(\mathrm{X}=\mathrm{N} \text { and } \mathrm{C})^{2,39}$ are metals. There is no anomaly indicative of a structural or electronic transition such as superconductivity in the resistivity curve of $\operatorname{SnXCo}_{3}(\mathrm{X}=\mathrm{N}$ and $\mathrm{C}$ ) above $2 \mathrm{~K}$. 

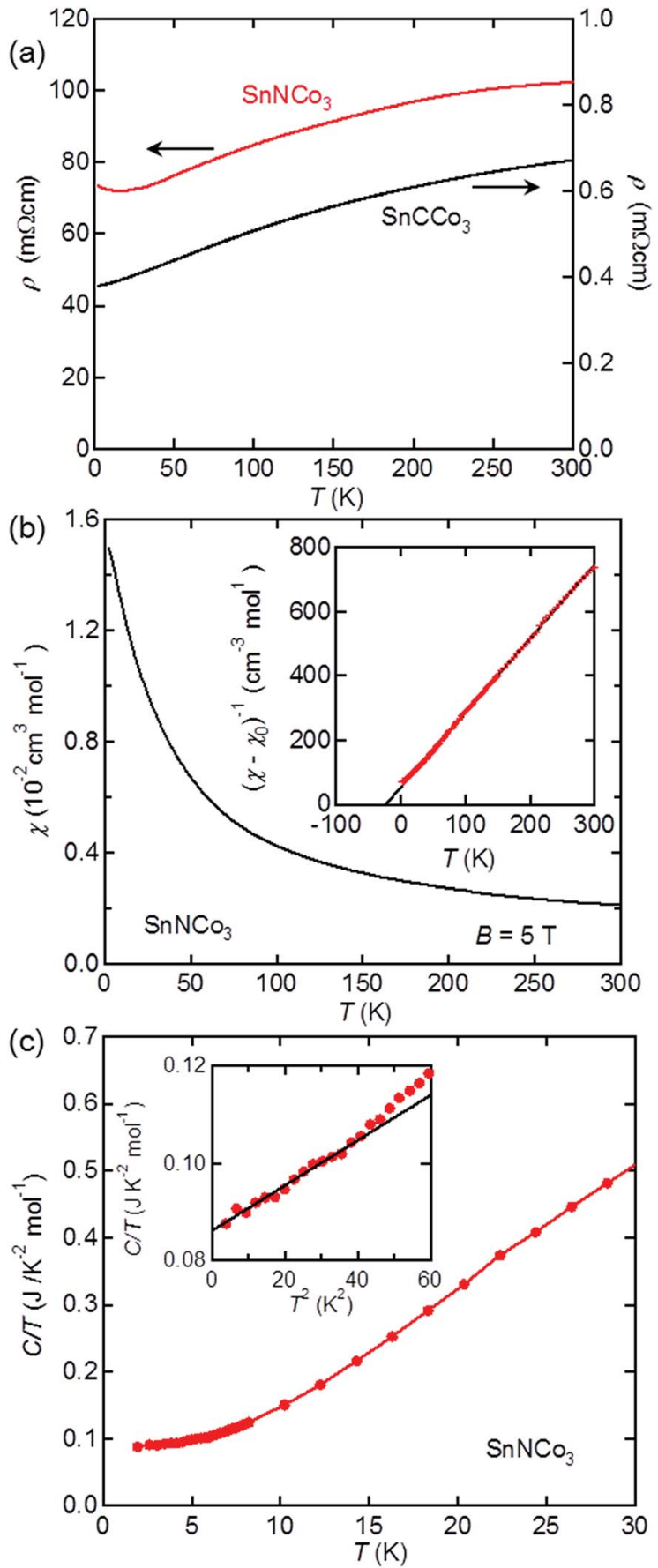

Fig. 5 Temperature dependences of the (a) resistivity, (b) magnetic susceptibility, and (c) heat capacity of $\mathrm{SnNCO}_{3}$. The resistivity data of $\mathrm{SnCCO}_{3}$ synthesised in this study are also shown in (a). The inset in (b) shows the inverse of the susceptibility after the subtraction of the temperature-independent term (red dots) with a Curie-Weiss fit indicated by the solid black line. The dependence of $C / T$ on $T^{2}$ is shown in the inset of (c).

$\mathrm{SnNCo}_{3}$ is paramagnetic down to $2 \mathrm{~K}$ with no anomaly indicative of magnetic transition in the temperature dependence of the magnetic susceptibility (Fig. 5(b)). The isothermal magnetic susceptibility at $300 \mathrm{~K}$ exhibits linear dependence on the applied field, which confirms the absence of a ferromagnetic component originating from impurity phases such as elemental Co. As shown in Fig. 5(b), the magnetic susceptibility rapidly increases with the decrease in temperature, which follows the Curie-Weiss behaviour expected for a localised magnet or correlated metal: $\chi=\left(T-\Theta_{\mathrm{W}}\right) / C+\chi_{0}$, where $C$ is the Curie constant, $\Theta_{\mathrm{W}}$ is the Weiss temperature, and $\chi_{0}$ is a temperature-independent constant. Fitting the data between 20 and 300 K yields $C=0.428(2) \mathrm{cm}^{3} \mathrm{~K} \mathrm{~mol}^{-1}, \Theta_{\mathrm{W}}=-22.3(2) \mathrm{K}$, and $\chi_{0}=7.68(11) \times 10^{-4} \mathrm{~cm}^{3} \mathrm{~mol}^{-1}$. The effective magnetic moment derived from the Curie constant is $\mu_{\text {eff }}=1.85 \mu_{\mathrm{B}}$ per formula unit including three Co ions, which is significantly smaller than expected for insulating Co magnets or ferromagnetic Co metal. Therefore, $\mathrm{SnNCo}_{3}$ is a strongly correlated itinerant magnet with dominant antiferromagnetic interactions, where a narrow band attributed to d-orbitals with a large density of states (DOS) is located at the Fermi energy $\left(E_{\mathrm{F}}\right)$.

The high DOS at the $E_{\mathrm{F}}$ in $\mathrm{SnNCo}_{3}$ was demonstrated by heat capacity measurements. As shown in Fig. 5(c), the lowtemperature heat capacity data between 2 and $6 \mathrm{~K}$ are well fitted to the equation: $C / T=\gamma+\beta T^{2}$, which yields $\gamma=86.1(4) \mathrm{mJ}$ $\mathrm{K}^{-1} \mathrm{~mol}^{-2}$ and $\beta=0.465(18) \mathrm{mJ} \mathrm{K}^{-1} \mathrm{~mol}^{-4}$. The finite electronic specific heat coefficient $\gamma$ indicates the existence of a Fermi surface in $\mathrm{SnNCo}_{3}$, which is consistent with the metallic resistivity. The $\gamma$ value is significantly higher than those of a conventional metal and related compounds such as $\operatorname{SnCCo}_{3}(\gamma$ $\left.=40.51(2) \mathrm{mJ} \mathrm{K}^{-2} \mathrm{~mol}^{-1}\right)^{16}$ and $\mathrm{ZnNNi}_{3}\left(\gamma=13 \mathrm{~mJ} \mathrm{~K}^{-2} \mathrm{~mol}^{-1}\right){ }^{2}$ The large $\gamma$ further confirms the correlated metallic state with high DOS at the $E_{\mathrm{F}}$ realised in $\mathrm{SnNCo}_{3}$. The Debye temperature of $\mathrm{SnNCO}_{3}$ calculated from the coefficient $\beta$ is $\Theta_{\mathrm{D}}=275(3) \mathrm{K}$, which is close to those of related anti-perovskite-type compounds $\mathrm{ZnN}_{y} \mathrm{Ni}_{3}\left(\Theta_{\mathrm{D}}=336 \mathrm{~K}\right)$ and $\mathrm{CdCNi}_{3}\left(\Theta_{\mathrm{D}}=352 \mathrm{~K}\right)^{2}$.

According to first-principle calculations for Co-based antiperovskite-type nitrides, ${ }^{\mathbf{4 2}}$ the hybridised Co-3d and $\mathrm{N}-2 \mathrm{p}$ electrons dominate the DOS around the $E_{\mathrm{F}}$, and thus determine their physical properties. The less-extended Co $3 \mathrm{~d}$ orbitals yield a narrow band with high DOS, which probably occurs in $\mathrm{SnNCo}_{3}$ in an enhanced manner. In such a situation, the magnetic instability associated with high DOS is expected. ${ }^{43}$ Even a small perturbation such as a physical or chemical pressure or chemical doping could significantly change the properties of $\mathrm{SnNCo}_{3}$, which should be investigated in subsequent studies.

\section{Reaction mechanism in the melamine method}

The reaction conditions of the melamine method in this study were compared to those for the syntheses of binary nitrides and carbides in previous studies. ${ }^{33,38}$ The reaction temperatures for the binary systems are often higher than that in this study. For example, the binary $3 \mathrm{~d}$-transition-metal nitride $\mathrm{VN}$ was synthesised at $1000{ }^{\circ} \mathrm{C}$ from $\mathrm{V}_{2} \mathrm{O}_{5}$ and melamine at a ratio of $\mathrm{V}_{2}$ $\mathrm{O}_{5}: \mathrm{C}_{3} \mathrm{H}_{6} \mathrm{~N}_{6}=1: 5 .{ }^{33} \mathrm{~A}$ higher reaction temperature of $1200{ }^{\circ} \mathrm{C}$ and higher melamine content $\left(\mathrm{V}_{2} \mathrm{O}_{5}: \mathrm{C}_{3} \mathrm{H}_{6} \mathrm{~N}_{6}=1: 8\right)$ are needed for the synthesis of $\mathrm{VC}^{38}$ The reaction for the $4 \mathrm{~d}$ nitride $\mathrm{NbN}$ was performed with a nominal composition of $\mathrm{Nb}_{2} \mathrm{O}_{5^{-}}$ $: \mathrm{C}_{3} \mathrm{H}_{6} \mathrm{~N}_{6}=1: 3$ and reaction temperature of $750{ }^{\circ} \mathrm{C}$, while that for the carbide counterpart $\mathrm{NbC}$ was performed at $\mathrm{Nb}_{2} \mathrm{O}_{5}: \mathrm{C}_{3^{-}}$ $\mathrm{H}_{6} \mathrm{~N}_{6}=1: 3$ and $1100{ }^{\circ} \mathrm{C} .{ }^{33,38}$ In contrast, the ternary nitrides and carbides in this study were obtained at approximately 600 
and $1000{ }^{\circ} \mathrm{C}$, respectively. On the other hand, the ammonolysis for $\mathrm{ZnNNi}_{3}$ was performed at $600{ }^{\circ} \mathrm{C}^{2}{ }^{2}$ These characteristics indicate that the anti-perovskite-type compounds are thermodynamically stable and can be synthesised at relatively low temperatures with the aid of an appropriate nitrogen source. It is worth noting that the reaction between $\mathrm{Co}_{2} \mathrm{O}_{3}$ or $\mathrm{Ni}_{2} \mathrm{O}_{3}$ and melamine in the absence of $\mathrm{Zn}$ or $\mathrm{Sn}$ does not form a binary nitride or carbide but reduces the oxides to elemental metals. ${ }^{38}$ The formation of the thermodynamically stable ternary compound may promote nitridation or carbidation reactions, which are not possible for the binary systems.

In the case of binary nitrides and carbides, carbides are typically obtained at higher nominal melamine contents and higher temperatures. ${ }^{33,38} \mathrm{~A}$ similar trend is observed in the case of ternary systems. $\mathrm{ZnCNi}_{3}$ and $\mathrm{SnCCo}_{3}$ were synthesised at higher melamine contents and reaction temperatures than those of the corresponding nitrides. We speculate that at high temperatures the formation of a stable $\mathrm{N}_{2}$ molecule reduces the reactive $\mathrm{N}$ species and thus terminates the nitridation so that carbidation alternatively occurs.

The nitrogen-source melamine polymerises, releasing $\mathrm{NH}_{3}$ gas and producing intermediate phases such as melam $\left(\mathrm{C}_{6} \mathrm{H}_{9} \mathrm{~N}_{11}\right)$, melem $\left(\mathrm{C}_{6} \mathrm{H}_{6} \mathrm{~N}_{10}\right)$, and melon $\left(\mathrm{C}_{18} \mathrm{H}_{9} \mathrm{~N}_{27}\right)$. Finally, graphitic carbon nitride $\left(\mathrm{g}-\mathrm{C}_{3} \mathrm{~N}_{4}\right)$ is formed below $520{ }^{\circ} \mathrm{C}$. Above $600{ }^{\circ} \mathrm{C}, \mathrm{g}-\mathrm{C}_{3} \mathrm{~N}_{4}$ decomposes to reactive radical species such as $\mathrm{C}_{3} \mathrm{~N}_{3}{ }^{+}, \mathrm{C}_{2} \mathrm{~N}_{2}{ }^{+}$, and $\mathrm{C}_{3} \mathrm{~N}_{2}{ }^{+} \cdot{ }^{35-37}$ As many chemical species are involved in the reaction process, the mechanism of nitridation and carbidation by melamine is not entirely understood. It is speculated that there are three reaction steps: reduction of oxide to metal, nitridation of the metal, and carbidation of the nitride. The reduction of oxides must occur at the first step as several transition-metal oxides are not transformed into nitrides but only reduced to elemental metals by the melamine reaction. The other two steps have been confirmed by preparations of carbides from reactions between elemental metals and melamine, and between nitride and melamine, respectively. ${ }^{38}$ This study shows that the carbides can be synthesised by the reaction between nitrides and melamine at high temperatures. Therefore, it seems that a similar reaction process is involved in the production of ternary compounds. For nitridation, it is suggested that the $\mathrm{NH}_{3}$ gas released from melamine is important in the reaction process. For the binary nitrides, another synthetic route through the reaction between oxides and $\mathrm{g}-\mathrm{C}_{3} \mathrm{~N}_{4}$ has been investigated. ${ }^{44} \mathrm{~A}$ comparison between the melamine reaction and $\mathrm{g}-\mathrm{C}_{3} \mathrm{~N}_{4}$ reaction will reveal the role of the $\mathrm{NH}_{3}$ gas in the reaction mechanism.

Finally, we compare the reaction conditions between the melamine method and the previously reported synthesis. $\mathrm{ZnNNi}_{3}$ was obtained by heating a mixture of elemental $\mathrm{Zn}$ and $\mathrm{Ni}$ in ammonia gas at $600{ }^{\circ} \mathrm{C}$ in the previous synthesis. ${ }^{2}$ The same reaction temperature was employed for $\mathrm{ZnNNi}_{3}$ in the melamine method, which implies that ammonia gas released from melamine plays a key role in the formation of nitrides. On the other hand, the carbides $\mathrm{ZnCNi}_{3}$ (ref. 39) and $\mathrm{SnCCo}_{3}$ (ref. 16) were synthesized by solid-state reaction using carbon powder in an inert atmosphere above $800{ }^{\circ} \mathrm{C}$. In the melamine method, a lower temperature of $650{ }^{\circ} \mathrm{C}$ and high temperature of
$1000{ }^{\circ} \mathrm{C}$ were employed, respectively. In these reactions, amorphous carbon likely reacted with metals to produce the carbides, which were generated from melamine in the case of the melamine method.

\section{Conclusions}

In summary, we employed nitridation and carbidation reactions using melamine in the syntheses of ternary carbides and nitrides with the anti-perovskite-type structures. By tuning the synthetic conditions such as nominal melamine content and reaction temperature, $\mathrm{ZnNNi}_{3}$ and $\mathrm{ZnCNi}_{3}$ were selectively prepared. Moreover, this method enabled the synthesise of a new compound, $\mathrm{SnNCo}_{3}$. Measurements of the physical properties showed that $\mathrm{SnNCo}_{3}$ is a correlated metal with a high DOS at $E_{\mathrm{F}}$. Our findings demonstrate that the melamine method is useful in the synthesis of complex carbides and nitrides, and provides a new route to study novel functional materials.

\section{Conflicts of interest}

There are no conflicts to declare.

\section{Acknowledgements}

This work was partly supported by Japan Society for the Promotion of Science (JSPS) KAKENHI Grant Number JP15K17695, Yazaki Memorial Foundation for Science and Technology, and by Core-to-Core Program (A) Advanced Research Networks.

\section{Notes and references}

1 T. He, Q. Huang, A. P. Ramirez, Y. Wang, K. A. Regan, N. Rogado, M. A. Hayward, M. K. Haas, J. S. Slusky, K. Inumara, H. W. Zandbergen, N. P. Ong and R. J. Cava, Nature, 2001, 411, 54-56.

2 M. Uehara, A. Uehara, K. Kozawa and Y. Kimishima, J. Phys. Soc. Japan, 2009, 78, 033702.

3 M. Uehara, A. Uehara, K. Kozawa, T. Yamazaki and Y. Kimishima, Phys. C Supercond., 2010, 470(suppl.), S688S690.

4 K. Takenaka and H. Takagi, Appl. Phys. Lett., 2005, 87, 1-3. 5 K. Takenaka, K. Asano, M. Misawa and H. Takagi, Appl. Phys. Lett., 2008, 92, 1-4.

6 R. Huang, L. Li, F. Cai, X. Xu and L. Qian, Appl. Phys. Lett., 2008, 93, 91-94.

7 T. Hamada and K. Takenaka, Cit. J. Appl. Phys., 2011, 109, 7309.

8 K. Kamishima, T. Goto, H. Nakagawa, N. Miura, M. Ohashi, N. Mori, T. Sasaki and T. Kanomata, Phys. Rev. B, 2000, 63, 024426.

9 Y. B. Li, W. F. Li, W. J. Feng, Y. Q. Zhang and Z. D. Zhang, Phys. Rev. B, 2005, 72, 024411.

10 B. S. Wang, P. Tong, Y. P. Sun, L. J. Li, W. Tang, W. J. Lu, X. B. Zhu, Z. R. Yang and W. H. Song, Appl. Phys. Lett., 2009, 95, 222509. 
11 Y. Sun, C. Wang, Y. Wen, K. Zhu and J. Zhao, Appl. Phys. Lett., 2007, 91, 231913.

12 K. Asano, K. Koyama and K. Takenaka, Appl. Phys. Lett., 2008, 92, 161909.

13 T. Tohei, H. Wada and T. Kanomata, J. Appl. Phys., 2003, 94, 1800-1802.

14 M.-H. Yu, L. H. Lewis and A. R. Moodenbaugh, J. Appl. Phys., 2003, 93, 10128-10130.

15 B. S. Wang, P. Tong, Y. P. Sun, X. Luo, X. B. Zhu, G. Li, X. D. Zhu, S. B. Zhang, Z. R. Yang, W. H. Song and J. M. Dai, EPL, 2009, 85, 47004.

16 S. Lin, P. Tong, B. Wang, J. Lin, Y. Huang and Y. Sun, Inorg. Chem., 2014, 53, 3709-3715.

17 Y. Okamoto, A. Sakamaki and K. Takenaka, J. Appl. Phys., 2016, 119, 1-6.

18 E. O. Chi, W. S. Kim and N. H. Hur, Solid State Commun, 2001, 120, 307-310.

19 S. G. Ebbinghaus, H. P. Abicht, R. Dronskowski, T. Müller, A. Reller and A. Weidenkaff, Prog. Solid State Chem., 2009, 37, 173-205.

20 L. H. Lewis, D. Yoder, A. R. Moodenbaugh, D. A. Fischer and M.-H. Yu, J. Phys. Condens. Matter, 2006, 18, 1677-1686.

21 T. Scholz and R. Dronskowski, Inorg. Chem., 2015, 54, 88008807.

22 P. R. Slater, J. Fluor. Chem., 2002, 117, 43-45.

23 F. J. Berry, X. Ren, R. Heap, P. Slater and M. F. Thomas, Solid State Commun, 2005, 134, 621-624.

24 Y. Kobayashi, M. Tian, M. Eguchi and T. E. Mallouk, J. Am. Chem. Soc., 2009, 131, 9849-9855.

25 D. Hirai, E. Climent-Pascual and R. J. Cava, Phys. Rev. B: Condens. Matter Mater. Phys., 2011, 84, 174519.

26 D. Hirai, O. Sawai, T. Nunoura and Z. Hiroi, J. Fluor. Chem., 2018, 209, 43-48.

27 D. Ostermann, H. Jacobs and B. Harbrecht, Zeitschrift für Anorg. und Allg. Chemie, 1993, 619, 1277-1282.

28 N. Arumugam, A. Hönnerscheid and M. Jansen, Zeitschrift für Anorg. und Allg. Chemie, 2003, 629, 939-941.
29 A. Gomathi, S. Reshma and C. N. R. Rao, J. Solid State Chem., 2009, 182, 72-76.

30 A. Gomathi, A. Sundaresan and C. N. R. Rao, J. Solid State Chem., 2007, 180, 291-295.

31 A. Gomathi and C. N. R. Rao, Mater. Res. Bull., 2006, 41, 941947.

32 A. Gomathi, Mater. Res. Bull., 2007, 42, 870-874.

33 H. Zhao, M. Lei, X. Chen and W. Tang, J. Mater. Chem., 2006, 16, 4407.

34 ChemIDplus - 108-78-1 - JDSHMPZPIAZGSV-UHFFFAOYSA$\mathrm{N}$ - Melamine - Similar structures search, synonyms, formulas, resource links, and other chemical information, https://chem.nlm.nih.gov/chemidplus/rn/108-78-1, accessed 23 July 2018.

35 B. Jürgens, E. Irran, J. Senker, P. Kroll, H. Müller and W. Schnick, J. Am. Chem. Soc., 2003, 125, 10288-10300.

36 M. Groenewolt and M. Antonietti, Adv. Mater., 2005, 17, 1789-1792.

37 B. V. Lotsch and W. Schnick, Chem. - A Eur. J., 2007, 13, 4956-4968.

38 M. Lei, H. Z. Zhao, H. Yang, B. Song and W. H. Tang, J. Eur. Ceram. Soc., 2008, 28, 1671-1677.

39 M.-S. Park, J. Giim, S.-H. Park, Y. W. Lee, S. I. Lee and E. J. Choi, Supercond. Sci. Technol., 2004, 17, 274.

40 P. L'Héritier, D. Fruchart, R. Madar, R. Fruchart and H. P. J. Wijn, Crystallographic properties of $\operatorname{M}\{c\} X M\{f\}\{3\}$ compounds: datasheet from Landolt-Börnstein - Group III Condensed Matter, Alloys and Compounds of d-Elements with Main Group Elements. Part 2 in SpringerMaterials, Springer-Verlag, Berlin Heidelberg, 1988, vol. 19C.

41 L. Pauling, J. Am. Chem. Soc., 1947, 69, 542-553.

42 Y. Medkour, A. Roumili, D. Maouche and M. Maamache, Solid State Commun, 2011, 151, 1916-1919.

43 D. Shao, W. Lu, P. Tong, S. Lin, J. Lin and Y. Sun, J. Phys. Soc. Japan, 2014, 83, 054704.

44 H. Zhao, M. Lei, X. Yang, J. Jian and X. Chen, J. Am. Chem. Soc., 2005, 127, 15722-15723. 